\title{
DISTRIBUTED MULTIACCESS IN HIERARCHICAL COGNITIVE RADIO NETWORKS
}

\author{
Shiyao Chen and Lang Tong \\ School of ECE, Cornell University, Ithaca, NY 14853 \\ Email: $\{$ sc933,1t35\}@cornell.edu
}

\begin{abstract}
The problem of distributed multiuser medium access of a hierarchical cognitive radio network with multiple primary and secondary users is considered. Each primary user has a licensed channel; it allows access by secondary cognitive users under certain collision constraints. The secondary cognitive users seek to capture transmission opportunities while avoiding collisions with primary and other secondary users.

Under tight collision constraints, we establish the maximum throughput region achievable by distributed multiaccess of secondary users when the number of secondary users is less than the number of primary channels using a scheme referred to as the Distributed Orthogonal and Periodic Sensing with Memoryless Access (DOPS-MA). Packet-level simulations are used to validate the performance prediction.
\end{abstract}

Index Terms - Cognitive radio networks, dynamic spectrum access, opportunistic multiaccess.

\section{INTRODUCTION}

In a hierarchical cognitive network [1] with the coexistence of primary users (PUs) and cognitive users (CUs), the PUs are owners of the spectrum and transmit over designated channels whenever they have packets in their queues. The CUs, on the other hand, have lower access priority and try to transmit in channels and at times when the PUs are not present. The CUs can sense channels for available opportunities and their transmissions are subject to interference constraints imposed by the PUs.

We consider the problem of distributed multiaccess of $N$ primary channels by $K \leq N$ CUs in the MAC layer. By distributed multiaccess we mean that there is no central controller to coordinate access for the CUs, and the CUs cannot communicate among themselves. This requirement makes the problem significantly more challenging as collisions among CUs are inevitable as they search individually for transmission opportunities.

The problem of determining maximum throughput region for multiple CUs can be formulated as a constrained Markov

This work is supported in part by the National Science Foundation under Contract CCF-1018115 and the Army Research Office MURI Program under award W911NF-08-1-0238. decision process with multiple distributed decision makers, which is in general intractable. It was shown earlier, however, that when the collision constraints imposed by the PUs are below certain thresholds (as functions of channel parameters), a policy referred to as Orthorgonalized Periodic Sensing with Memoryless Access (OPS-MA) achieves the maximum throughput region. The OPS-MA policy proposed earlier, however, requires a nontrivial pre-arrangement in sensing phases for the CUs. In particular, each $\mathrm{CU}$ is assigned a pre-determined sensing phase, which orthorgonalizes sensing and transmissions of CUs. Such pre-arrangement may require centralized coordination. A natural question therefore is whether the throughput region achieved by OPS-MA can be achieved in a pure distributed manner, which precludes prearrangement.

\subsection{Summary of Results and Contributions}

In this paper we obtain the maximum throughput region of the cognitive access network without pre-arrangement. We show in Section 3 that under tight collision constraints, DOPS-MA achieves all the interior points of the maximum throughput region with pre-arrangement. This result generalizes that in [2] where pre-arranged cognitive access is assumed and demonstrates that the performance loss by dropping the prearrangement is negligible.

We obtain consistent detection of available sensing phases and collision among the CUs. The proposed multiaccess scheme DOPS-MA with phase and collision detection removes the requirement of pre-arrangement, realizes the selfconfiguration of the CUs (i.e., pinning down to an available sensing phase by each CU itself without any central coordination), and accommodates asynchronous arrival of the CUs (assuming the time slots are synchronized in the network).

\subsection{Related Work}

The problem of single user access of hierarchical cognitive networks was first considered in [3] under a decision theoretic framework. Under a slotted transmission system, the PU's channels are modeled as independent discrete time Markov chains with on and off states. The optimal sensing policy is shown to be a myopic policy $[4,5]$ for i.i.d. and positively 
correlated Markov chains. For unslotted primary user systems, a more appropriated model for the PUs is the continuous time on-off Markov process, and the single user access of such cognitive network was first considered in $[6,7]$. The PS-MA policy is shown to be optimal for the single CU case in [8].

For multiuser cognitive access, the problem is considerably more challenging. For continuous time primary traffic, the authors of [2] gave the first characterization of throughput and effective bandwidth regions under the assumption that pre-arrangement can be made among CUs. An ALOHA style random access strategy was considered in [9].

There has been effort in formulating multiuser cognitive access from a distributed learning perspective [10-12]. These formulations are for slotted primary systems with independent Bernoulli primary traffic patterns with unknown probability of channel availability. The problem considered in this paper is very different from that in [10-12]. In our formulation, the primary users follow continuous time Markov channels with known parameters. Thus the learning aspect of the problem is not with respect to channel parameters but with respect to behaviors of other users. In particular, our channel and access models are more complex and general than that in [10-12] with the assumption that model parameters are known.

\section{NETWORK MODEL}

\subsection{Models for Primary and Cognitive Users}

We assume an $N$ primary user hierarchical network. Each primary user transmits independently from others on its designated channel. The occupancy of channel $i$ is modeled by a continuous time Markov busy-idle process with busy (1) and idle (0) periods exponentially distributed with means $\mu_{i}^{-1}$ and $\lambda_{i}^{-1}$, respectively. See [6] for an experimental justification. The stationary distribution for idle (0) state under the continuous time Markov channel model is given by $v_{i}(0)=\mu_{i} /\left(\mu_{i}+\lambda_{i}\right)$.

The cognitive users follow a slotted sensing and transmission structure with slot length $T$ generalized from the bluetooth access protocol. Each CU senses one channel at a time and decides whether to transmit. Channel sensing is assumed perfect. Each $\mathrm{CU}$ acts independently and initially has no knowledge of the presence of other CUs. When a CU accesses a channel in slot $t$, the $\mathrm{CU}$ collects unit reward if the channel is idle throughout slot $t$ and no other CUs access the same channel. Our results applies to the case the number $K$ of CUs is less than $N$-the number of primary users.

\subsection{Throughput and Interference Constraint}

Being secondary users, the CUs have to transmit under collision constraints imposed by PUs, i.e., the overall collision caused by the CUs to PU $i$ should be bounded by a collision constraint parameter $\gamma_{i}$. The collision for PU $i$ is the fraction of the collided time out of the total primary transmission time. Specifically, the collision for PU $i$ is defined to be the fraction of the collided slots out of the slots fully or partially used by PU $i$ (due to the continuous time transmission process assumed for PUs). Equivalently, the overall collision on channel $i$ is given by the infinite horizon average collision scaled by the reciprocal of the steady state probability of PU $i$ transmitting in a certain slot, as given below,

$$
C_{i}=\frac{1}{1-v_{i}(0) e^{-\lambda_{i} T}} \lim _{n \rightarrow \infty} \frac{1}{n} \mathbb{E} \sum_{t=1}^{n} 1_{\{\text {collide PU } i \text { in slot } t\}}
$$

We measure the system performance with the throughput of the CUs. Denote by $R_{t}^{(k)}$ the reward that CU $k$ collects in slot $t$. The throughput of CU $k$ is defined by the infinite horizon average reward, i.e., $J^{(k)}=\lim _{n \rightarrow \infty} \frac{1}{n} \mathbb{E} \sum_{t=1}^{n} R_{t}^{(k)}$.

Given collision parameter $\gamma=\left(\gamma_{1}, \cdots, \gamma_{N}\right)$, the set of admissible policies $\boldsymbol{\Pi}$ is $\left\{\boldsymbol{\pi}=\left(\pi_{1}, \ldots, \pi_{K}\right): C_{\boldsymbol{\pi}, i} \leq\right.$ $\left.\gamma_{i}, i=1, \ldots, N\right\}$ where $\pi_{k}$ is the sensing and transmission policy of CU $k$. The throughput vector of policy $\boldsymbol{\pi}$ is $\mathbf{J}_{\boldsymbol{\pi}}=\left(J_{\boldsymbol{\pi}}^{(1)}, \ldots, J_{\boldsymbol{\pi}}^{(K)}\right)$ and the throughput region is $\mathscr{J}=\bigcup_{\boldsymbol{\pi} \in \boldsymbol{\Pi}} \mathbf{J}_{\boldsymbol{\pi}}$.

\subsection{Throughput Region with Pre-arrangement}

It was established in [2] that OPS-MA achieves the maximum throughput region under tight collision constraints assuming pre-arrangement of the sensing phases.

$\bigcirc$ Idle sensing for CU $1 \square$ Busy sensing for CU 1

$\checkmark$ Idle sensing for CU $2 \triangle$ Busy sensing for CU 2

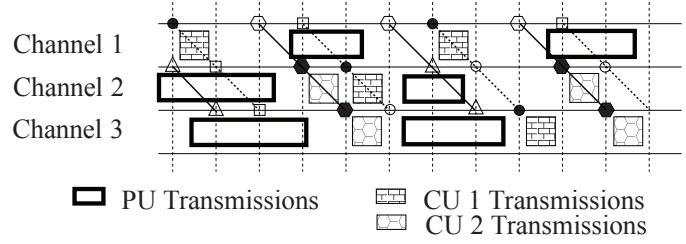

Fig. 1. Illustration of OPS-MA policy. Open circle: CUs decide not to transmit. Filled circle: CUs decide to transmit.

The sensing policy of OPS-MA is orthogonal and periodic. Each CU senses the $N$ channels in an increasing order, e.g., starting from a specific time slot, the two sequences of channel indices $1,2,3, \ldots, N$ and $2,3, \ldots, N, 1$ are two different sensing orders and we term the sensing order as sensing phase. There are total $N$ different sensing phases and in the case with sensing phase pre-arrangement the $K$ CUs sense the channels using pre-arranged different sensing phases, which precludes collisions among the CUs. The transmission policy of OPS-MA is memoryless. If channel $i$ is sensed to be idle by CU $k, \mathrm{CU} k$ transmits in channel $i$ w.p. $\mu_{i}^{(k)}$. The $\mu_{i}^{(k)}$, s are determined by the collision parameter $\gamma$. We refer to [2] for a detailed description. 


\section{MAXIMUM THROUGHPUT REGION OF DISTRIBUTED ACCESS}

\subsection{DOPS-MA}

We now consider the case without pre-arrangement among CUs. We propose a generalization of OPS-MA called Distributed Orthogonal and Periodic Sensing with Memoryless Access (DOPS-MA). DOPS-MA assumes that all CUs use the periodic sensing and memoryless transmission protocol except that each user senses channel 1 (or any fixed channel) at a different time. We define the time $(\bmod N)$ that a $\mathrm{CU}$ senses channel 1 to be the sensing phase of the CU. If all CUs have different sensing phases, the result in [2] guarantees that the maximum throughput region is achieved.

The key ingredient of DOPS-MA is a (sensing) phase detection scheme so that, when the algorithm is applied individually by CUs, the CUs' sensing and transmissions can be orthogonalized by the (sensing) phase detection mechanism. The key is that the phase detection algorithm must allow each $\mathrm{CU}$ to identify an unused phase quickly enough so that the overall throughput does not suffer. The difficulty is that, when a slot on a channel is being used, the CU cannot easily distinguish whether it is by a primary user or by another CU. Similarly, when the $\mathrm{CU}$ transmits and collides with some other user, it does not know for sure the collision is caused by another CU or by a PU. Such uncertainties make the problem nontrivial.

In DOPS-MA, each CU follows a two-stage protocol. In the first stage, the CU performs phase detection until it identifies a candidate of unoccupied phase. The identification can of course be incorrect; it is characterized by the receiver operating characteristics of the detector. In the second stage, the CU uses the identified phase to sense and transmit while monitoring its collision behavior with a CU collision detector. The $\mathrm{CU}$ will either evacuate and go back to stage 1, if the CU collision detector claims collision with another $\mathrm{CU}$, i.e., two or more CUs in stage 2 are sensing and transmitting in the same phase, or stay on the same phase otherwise. Therefore, each $\mathrm{CU}$ in the network is either in stage 1 or stage 2 . The fraction of time a CU spends in stage 1 and stage 2 are determined by the performance of the sensing phase detector as well as the CU collision detector.

\subsection{Sensing Phase Detection and CU Collision Detection}

We now considers the specifics of sensing phase detection and CU collision detection. Assume that each time slot $T$ is divided into $L$ mini slots. The CUs in stage 1 devote all $L$ mini slots in sensing while the CUs in stage 2 spend the first mini slot in sensing and try to transmit in the rest $L-1$ mini slots if the sensing result is idle; both types spend the first mini slot in each slot sensing the channel, therefore no cognitive transmission in the first mini slot.
Since a stage $1 \mathrm{CU}$ does not know the transmission probability $\mu_{i}^{(k)}$ of the stage $2 \mathrm{CU}(\mathrm{s})$ already in the phase, we formulate the phase detection as a composite hypothesis test

$\mathcal{H}_{0}^{s}: \quad$ No CU in the phase vs.

$\mathcal{H}_{1}^{s}$ : Certain CU in the phase, transmitting w.p.

$$
\boldsymbol{\mu}=\left(\mu_{1}, \ldots, \mu_{N}\right), \mu_{i} \geq \mu_{\min }
$$

where $\mu_{\min }$ is the minimum transmission probability ever used by any CU.

The observation for available phase detection in one slot is $Y_{n} \in\{0$ (idle $), 1$ (busy) $\}^{L}$. If a stage $1 \mathrm{CU}$ follows a particular phase, the observations $\left\{Y_{n}^{i}\right\}$ from channel $i$ are independent of observations from other channels and the dynamics of the observation $\left\{Y_{n}^{i}\right\}$ can be shown to be a Markov chain under both hypotheses [13].

In order to detect collision with other stage $2 \mathrm{CU}(\mathrm{s})$ on the same phase, a stage $2 \mathrm{CU}$ collects the feedback of the transmissions (failure or success). Given an idle sensing result of channel $i$ in the first mini slot, the observations under $\mathcal{H}_{0}^{c}$ (No other CU accessing the phase) and $\mathcal{H}_{1}^{c}$ (Some other $\mathrm{CU}(\mathrm{s})$ accessing the phase with transmission probability $\hat{\boldsymbol{\mu}}$, $\left.\hat{\mu}_{i}>\hat{\mu}_{\min }\right)$ are given by

$$
Y^{i}= \begin{cases}0 \text { (fail) } & \text { w.p. } \mu_{i}\left(1-e^{-\lambda_{i} T}\right) \\ 1 \text { (success) } & \text { w.p. } \mu_{i} e^{-\lambda_{i} T} \\ e \text { (not transmitting) } & \text { w.p. } 1-\mu_{i}\end{cases}
$$

and

$Y^{i}= \begin{cases}0 \text { (fail) } & \text { w.p. } \mu_{i}\left(1-e^{-\lambda_{i} T}\left(1-\hat{\mu}_{i}\right)\right) \\ 1 \text { (success) } & \text { w.p. } \mu_{i} e^{-\lambda_{i} T}\left(1-\hat{\mu}_{i}\right) \\ e \text { (not transmitting) } & \text { w.p. } 1-\mu_{i} .\end{cases}$

For the phase detection, take the statistic $T^{s}(\boldsymbol{y})=$ $\sum_{i=1}^{N} \ln \frac{\pi_{i}^{\mu_{\min }}\left(y_{1}^{i}\right)}{\pi_{i}^{0}\left(y_{1}^{i}\right)}+\sum_{1 \leq k, l \leq 5} q_{k l}\left(\boldsymbol{y}^{i}\right) \ln \frac{P_{i, k l}^{\mu_{\min }}}{P_{i, k l}^{0}}$ where $\pi_{i}^{\mu_{\min }}$ and $\pi_{i}^{0}$ are the initial distribution under $\mathcal{H}_{1}^{s}$ with $\boldsymbol{\mu}=$ $\left(\mu_{\min }, \ldots, \mu_{\min }\right)$ and $\mathcal{H}_{0}^{s}, P_{i, k l}^{\mu_{\min }}$ and $P_{i, k l}^{0}$ are the $(k, l)$ th entries of the transition matrices of $Y_{n}^{i}$ under the two hypotheses, and $q_{k l}\left(\boldsymbol{y}^{i}\right)$ is the number of transitions from state $k$ to state $l$ in $\boldsymbol{y}^{i}$. For the collision detection take the statistic $T^{c}(\boldsymbol{y})=\sum_{i=1}^{N}\left[\#_{i} 1 \ln \left(1-\hat{\mu}_{\min }\right)+\#_{i} 0 \ln \frac{1-e^{-\lambda_{i} T}\left(1-\hat{\mu}_{\min }\right)}{1-e^{-\lambda_{i} T}}\right]$ where $\#_{i} 1$ and $\#_{i} 0$ are the number of success and failure feedbacks from channel $i$ respectively. $T^{s}(\cdot)$ and $T^{c}(\cdot)$ are the log likelihood ratio of the simple hypothesis tests $\mathcal{H}_{1}^{s}$ with $\boldsymbol{\mu}=\left(\mu_{\min }, \ldots, \mu_{\min }\right)$ vs. $\mathcal{H}_{0}^{s}$ and $\mathcal{H}_{1}^{c}$ with $\hat{\boldsymbol{\mu}}=\left(\hat{\mu}_{\min }, \ldots, \hat{\mu}_{\min }\right)$ vs. $\mathcal{H}_{0}^{c}$.

Lemma 1 (Consistency) With properly chosen threshold, the threshold test $\delta_{n}^{s}$ on the statistics $T^{s}(\boldsymbol{y})$ and $\delta_{n}^{c}$ on $T^{c}(\boldsymbol{y})$ are consistent for phase and collision detections, respectively, i.e., the miss detection and false alarm probabilities approach zero as $n \rightarrow \infty$.

Proof of Lemma 1 See [13]. 


\subsection{Optimality of DOPS-MA}

In DOPS-MA an incoming CU first senses a uniformly chosen phase for $M$ slots and comes to a decision whether the phase is available. If $\delta_{M}^{s}$ claims available, the CU transits to stage 2 and starts to access the phase.

In the process of accessing, a CU employs $\delta_{M}^{c}$ to detect collision with other $\mathrm{CU}(\mathrm{s})$ (caused by, e.g., miss detection of $\left.\delta_{M}^{s}\right)$. Whenever a collision with other $\mathrm{CU}(\mathrm{s})$ is claimed, the CU evacuates the current phase, transits to stage 1 and starts to sense a uniformly chosen phase. In the DOPS-MA policy, $M$ is the number of slots the detectors will monitor before making a decision, therefore larger $M$ leads to better detection performance.

The DOPS-MA scheme allows the arriving CUs to explore the available phases and avoid collisions among CUs. We have the following throughput region result for DOPSMA without pre-arrangement.

Theorem 1 (Optimality of DOPS-MA) Under collision constraints that

$$
\gamma_{i} \leq \frac{v_{i}(0)\left(1-\exp \left(-\lambda_{i} T\right)\right)}{N\left(1-v_{i}(0) \exp \left(-\lambda_{i} T\right)\right)}, \quad 1 \leq i \leq N,
$$

DOPS-MA achieves the maximum throughput region given by

$$
\mathscr{J}^{\prime}=\left\{\left(y_{1}, \ldots, y_{K}\right) \mid \sum_{k=1}^{K} y_{k}<\sum_{i=1}^{N} \phi_{i} g_{i} \gamma_{i}, y_{k}>0\right\},
$$

where $g_{i}=1-v_{i}(0) \exp \left(-\lambda_{i} T\right)$ and $\phi_{i}=\frac{\exp \left(-\lambda_{i} T\right)}{1-\exp \left(-\lambda_{i} T\right)}$.

Proof of Theorem 1 The key of the proof is the convergence of the stationary probability of the states where all the CUs are in stage 2 with different sensing phases as $M$ approaches $\infty$. See [13] for details.

Theorem 1 indicates that by using sensing phase detection and CU collision detection DOPS-MA achieves the interior of the throughput region obtained with pre-arrangement. We comment on the role of the parameter $M$, i.e., the number of slots the two detectors monitor before making a decision. Larger $M$ leads to better detection performance at the cost of making stage 1 (sensing for an unused phase) more expensive. However, since we are dealing with infinite horizon throughput, the effect of more costly stage 1 vanishes and larger $M$ yields throughput region closer to that of the prearranged setup.

\section{SIMULATION}

We give the simulation results for $N=K=2$. The channel parameters are $\mu=[1 / 1,1 / 1.43] \mathrm{ms}^{-1}, \lambda=[1 / 4.2,1 / 3.23] \mathrm{ms}^{-1}$, slot length $T=0.25 \mathrm{~ms}$, collision constraint parameter $\gamma=$ $[0.05,0.05]$, and horizon $10000 \mathrm{~ms}$. The simulated throughput region with different $M$ and that with pre-arrangement are shown in Fig. 2, which validates the statement in Theorem 1.

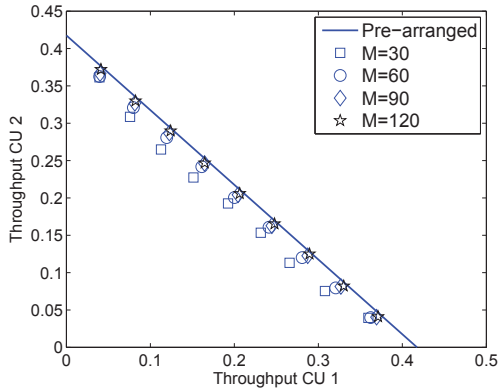

Fig. 2. Throughput region. Solid line: with pre-arrangement.

\section{REFERENCES}

[1] Q. Zhao and B. Sadler, "A Survey of Dynamic Spectrum Access,” IEEE Signal Processing Magazine, vol. 24, no. 3, 2007.

[2] S. Chen and L. Tong, "Multiuser cognitive access of continuous time Markov channels: maximum throughput and effective bandwidth regions," in Proc. UCSD ITA Workshop, 2010.

[3] Q. Zhao, L. Tong, A. Swami, and Y. Chen, "Decentralized cognitive MAC for opportunistic spectrum access in ad hoc networks: a POMDP framework," IEEE J. Select. Comm., vol. 25, no. 4, 2007.

[4] Q. Zhao, B. Krishnamachari, and K. Liu, "On myopic sensing for multi-channel opportunistic access: structure, optimality, and performance," IEEE Trans. Wireless Comm., 2008.

[5] S.H. Ahmad, M. Liu, T. Javidi, Q. Zhao, and B. Krishnamachari, "Optimality of myopic sensing in multi-channel opportunistic access," IEEE Trans. Inf. Theory, 2009.

[6] S. Geirhofer, L. Tong, and B. Sadler, "Cognitive medium access: constraining interference based on experimental models," IEEE J. Select. Comm., vol. 36, no. 2, 2008.

[7] Q. C. Zhao, S. Geirhofer, L. Tong, and B. Sadler, "Opportunistic spectrum access via periodic channel sensing," IEEE Trans. Signal Processing, vol. 36, no. 2, 2008.

[8] X. Li, Q. C. Zhao, X. Guan, and L. Tong, "Optimal cognitive access of Markovian channels under tight collision constraints," to appear in IEEE J. Select. Comm., acsp.ece.cornell.edu/papers/LiZhaoGuanTong11JSAC.pdf.

[9] S. Wang, J. Zhang, and L. Tong, "Delay analysis for cognitive radio networks with random access: a fluid queue view," in Proc. IEEE INFOCOM, 2010.

[10] L. Lai, H. Jiang, and V. Poor, "Medium Access in Cognitive Radio Networks: A Competitive Multi-armed Bandit Framework," in Proc. of IEEE Asilomar Conference on Signals, Systems, and Computers, 2008.

[11] K. Liu and Q. Zhao, "Distributed learning in multi-armed bandit with multiple players," IEEE Trans. Signal Processing, vol. 58 , no. $11,2010$.

[12] A. Anandkumar, N. Michael, and A. Tang, "Opportunistic spectrum access with multiple users: Learning under competition," in Proc. 2010 IEEE INFOCOM, 2010.

[13] S. Chen and L. Tong, "On distributed medium access of hierarchical cognitive networks," to be submitted to the IEEE Trans. Signal Processing. 